\title{
Prevalence, techniques and knowledge of rapid weight loss amongst adult british judo athletes: a questionnaire based study
}

\author{
Nikos Malliaropoulos ${ }^{1}$ \\ Shaan Rachid ${ }^{1}$ \\ Vasileios Korakakis ${ }^{2}$ \\ Stephen Andrew Fraser ${ }^{1}$ \\ Georgios Bikos ${ }^{3}$ \\ Nicola Maffulli ${ }^{1}$ \\ Manuela Angioi ${ }^{1}$ \\ 1 Queen Mary University of London, Centre for Sports \\ and Exercise Medicine, Mile End Hospital, London, UK \\ 2 Faculty of Physical Education and Sport Science, \\ University of Thessaly, Trikala, Greece \\ ${ }^{3}$ Euromedica-Arogi Rehabilitation Clinic, Thessaloniki, \\ Greece
}

Corresponding Author:

Nicola Maffulli

University of Salerno School of Medicine, Surgery and

Dentistry, Salerno, Italy

Queen Mary University of London,

Centre for Sports and Exercise Medicine

Mile End Hospital, Bancroft Road, London, E1 4DG

E-mail: n.maffulli@qmul.ac.uk

\section{Summary}

Background: No investigation has analysed the RWL approaches since new rules by the International Judo Federation (IJF) regarding weigh-ins have been implemented in international competitions in 2014. The current study analyses the prevalence, most common methods and knowledge surrounding the effects of rapid weight loss (RWL) amongst adult UK judo athletes. Additionally, it examines differences in rapid weight loss behaviour (RWLB) between gender, weight-class, competitive level, age RWL began and "high"/"Iow" knowledge athletes.

Methods: A previously validated questionnaire developed to assess RWL in judo athletes was modified by adding a knowledge section and revalidated for content. The questionnaire provided a RWLB score (higher score equated to more aggressive RWL) and a knowledge score (out of 10). 256 athletes (189 males and 66 females, aged 18-67 years) completed the questionnaire between February and April 2015. Unpaired $t$ test, one-way ANOVA-tests and Chi-squared tests were used to test differences of mean RWLB scores and prevalence between groups.
Results: The prevalence of RWL was $\mathbf{8 4} \%$. The most common methods of RWL were increased exercise and decreased food/fluid intake. The mean knowledge score was $6.2 \pm 2.8$, with most incorrect answers regarding physiological effects of RWL. No significant differences in RWLB scores were found between gender, "high"/"Iow" knowledge athletes or weight-classes. Statistically significant differences in RWLB scores were found between different competitive levels $(p=0.014)$ and age RWL began $(p<0.01)$ in males but not in females.

Conclusion: RWL is highly prevalent in the UK adult judo population and athletes have moderate knowledge surrounding its effects. In males, RWLB is most aggressive in elite level athletes and those that began RWL at a younger age. Therefore, updated rulings to target elite and youth level athletes should be implemented to reduce dangerous RWL.

Level of evidence: IV.

KEY WORDS: adult, martial arts, judo, weight-loss/ physiology, UK.

\section{Background}

Judo is the most popular Olympic combat sport practiced throughout the world ${ }^{1}$. In combat sports, competitors are grouped in weight classes to ensure more equitable matches, so that opponents of similar size and strength are paired against one another ${ }^{2}$.

Athletes in other combat sports, including wrestling ${ }^{3}$, taekwondo $^{4}$, jujitsu ${ }^{5}$ and karate ${ }^{5}$, use rapid weight loss (RWL) prior to competitions. In this way, competitors can enter at the top band of a lower weight class and therefore have a more favourable power to weight ratio. RWL impacts negatively several health parameters, including bone density ${ }^{6}$, muscular performance ${ }^{7,8}$, cardiovascular $^{9}$ and cognitive function ${ }^{10}$. Furthermore, RWL increases the chances of injury ${ }^{4}$, and therefore attempts have been made to reduce its prevalence in sports such as wrestling ${ }^{11}$.

Recent studies $5,12-14$ have revealed the extremely high prevalence of $89 \%$ of RWL is present among judo players. This is alarmingly higher than in other combat sports (e.g. jujitsu-56.8\%, taekwondo-63.3\%) ${ }^{5}$. The studies on RWL in judokas have methodological flaws, including mixing different combat athletes ${ }^{12,13}$, and including both child and adult judokas ${ }^{14}$. As of 2014, new rules by the International Judo Federation (IJF) regarding weigh-ins have been implemented in international 
competitions ${ }^{15}$. Judokas are now weighed the night before a competition, combined with random weigh-ins on the morning of the competition ${ }^{15}$. No investigation has analysed the RWL approaches since this ruling change. Additionally, education has been postulated as the solution to reduce the high RWL prevalence $2,14,16$. This is based on the assumption that knowledge regarding RWL is lacking in the judoka population, and education will reduce RWL prevalence. However, to our knowledge, these assumptions have never been verified.

\section{Objectives}

This questionnaire-based study explored the RWL trends amongst adult British judokas, with particular focus on prevalence, methods and knowledge surrounding the health effects of RWL by using a previously validated and successfully used questionnaire ${ }^{17}$.

We aimed to identify the different prevalence of RWL between judokas, recording gender, weight class and competitive level. We also aimed to identify the methods of RWL, and to assess the knowledge of athletes surrounding the effects of RWL.

Furthermore, we wished to uncover differences in rapid weight loss behaviour (RWLB) between genders, weight classes, competitive level, age when RWL began, and the knowledge that the athletes had regarding RWL and its effects.

\section{Materials/subjects and methods}

A previously validated questionnaire ${ }^{17}$ assessing weight loss behaviour in judokas was used. The questionnaire was composed of three main sections incorporating demographic/anthropometric measures, judo history and RWL history. From the RWL history section of the questionnaire, RWLB was defined through a numerical scale where the higher RWLB score the more aggressive the RWLB. The minimum score could be 0 , and had no upper limit. This score showed strong discriminant validity in distinguishing athletes who do and do not use RWL ${ }^{17}$. The questionnaire had been validated by 10 experts and displayed strong reproducibility (Intra-class Coefficient Correlation $>0.90)^{17}$.

For the purposes of the present study, questions assessing the judoka's knowledge were then designed and added on to the end of this existing questionnaire giving a total of 4 sections (Tab. I).

A literature review and a focus group were used to develop questions. Two independent reviewers performed the literature review (6/01/2015). Prominent topics that arose included the RWL effect on "cognition", "endocrine", "bone density", "cardiac accidents", "injury" and "performance".

The focus group consisted of five male judokas, ranging from regional to international level and aged between 21 50 from a London-based judo club. Potential questions from the literature review were presented to them. They

Table I. Outcome Measures of Questionnaire.

\begin{tabular}{ll}
\hline Section of Questionnaire & Outcome measure \\
\hline 1) Demographic information & Numerical data: age, weight, height \\
& Categorical: gender \\
2) Judo history & Numerical data: age began competing in judo \\
& Categorical data: competitive level \\
3) Weight history and RWL techniques* & Numerical data: amount of weight loss, number of times, age \\
& RWL began \\
& Categorical data: Whether they undertake RWL, weight loss \\
& methods used, weight class \\
4) Awareness of RWL effects (Knowledge based questions) & Numerical data: Knowledge score calculated out of 10
\end{tabular}

*This was used to calculate the RWL score, which quantified the aggressiveness of RWL behaviour based on a externally validated scoring system (15). The higher the score the more aggressive the RWL behaviour.

Table II. Panel of experts for content validation.

\begin{tabular}{ll}
\hline Expert & Experience \\
\hline Dietician & $\begin{array}{l}\text { Dietician to international judo athletes. 3 years experience } \\
\text { in judo } \\
\text { Judo Coach }\end{array}$ \\
$\begin{array}{ll}\text { Level } 3 \text { coach employed by British Judo Associated for } \\
\text { past } 6 \text { years. } 35 \text { years involvement in judo }\end{array}$ \\
Doctor & $\begin{array}{l}\text { Doctor to international judo athletes with consultant level } \\
\text { experience. } 30 \text { years involvement in judo }\end{array}$ \\
Elite Judo athlete & $\begin{array}{l}2^{\text {nd }} \text { dan in judo, competed for more than } 9 \text { years in national } \\
\text { and international competitions }\end{array}$ \\
\hline
\end{tabular}


Prevalence, techniques and knowledge of rapid weight loss amongst adult british judo athletes: a questionnaire based study

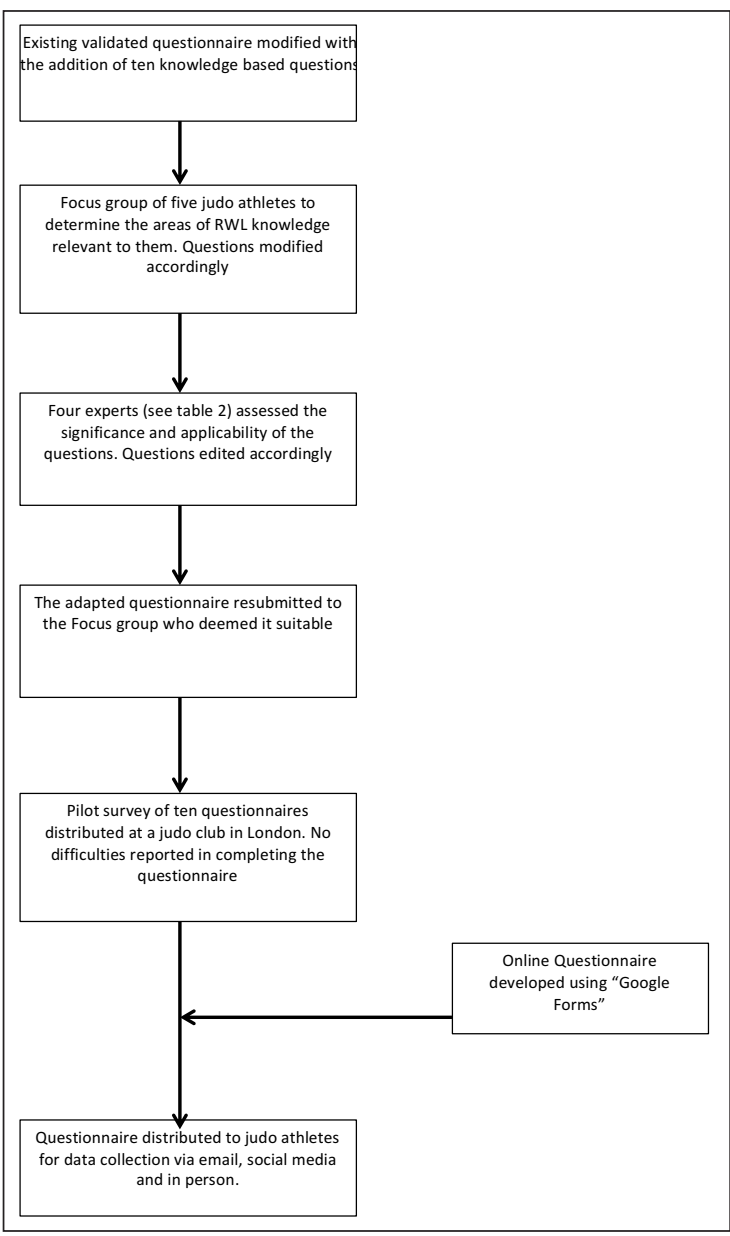

Figure 1. Flow Diagram of Validated Questionnaire development.

agreed that the health affects of RWL were significant areas, and also added awareness of diuretic use.

A panel of four experts (Tab. II) consisting of a dietician, a judo coach, a doctor and an elite judoka, all with a minimum of 3 years of experience in judo, evaluated the potential questions independently of each other. They reviewed the items for their relevance, and then convened to discuss the agreed questions. Redundant questions were eliminated. The questions were formulated to be unbiased and independent from each other, with the correct answer being determined from empirical evidence.

Ten categorical questions relating to the effects of RWL (answered as "yes"/"no"/"unsure") were formulated.

Each correct answer scored 1 point and used to quantify the judokas overall knowledge surrounding RWL (out of 10). Athletes scoring in the top and bottom decile would then be defined as "high" and "low" knowledge respectively.

The 10 questions formulated were resubmitted to the focus group, which then deemed the questionnaire suitable, and agreed that it would allow to define judokas' knowledge on RWL.

The 10 knowledge-based questions were subsequently added to the end of the previously validated questionnaire and then a pilot survey of 10 judokas at a London-based judo club was conducted to ensure usability of the questionnaire. Finally, an online edition of the questionnaire was developed (via "Google Forms") and distributed to the population (Fig. 1).

The study meets the ethical standards of MLTJ as described by Padulo et al. ${ }^{18}$.

A total of 255 participants (189 males and 66 females, 18-67 years) completed the questionnaire between February and April 2015. Male and female participants were included if they were over 18, had competed in an official competition within the last two years, and had a minimum of two years of competitive experience. Questionnaires with missing data and judokas under the age of 18 were excluded.

All athletes received a complete explanation of the objectives of the study. If they were willing to take part, they signed a consent form. The hard copy survey was conducted at two judo clubs in London. The online questionnaire was distributed via mailing lists of judo clubs and social media ("Facebook"). The online questionnaire also included a clear explanation of the objectives of the study and of the criteria that determined whether the participants were eligible to complete it. Consent was assumed if the questionnaire was completed. Queen Mary's University of London ethics committee granted full approval for this study.

Descriptive statistics were used for all numerical variables. Normal distribution of the RWL scores was assessed using the Kolmogorov-Smirnov test to guide parametric or non-parametric testing. The RWLB scores were normally distributed in female judokas only. Data were normalised using square-root transformation $^{19}$ since parametric testing is the most robust approach in analysing numerical data.

An unpaired $t$-test ${ }^{20}$ was performed to compare differences in RWLB scores (numerical data) between two independent groups (males with females and "High knowledge athletes" with "Low knowledge athletes"). One-way ANOVA 20 was used to compare RWLB scores between three or more independent variables including different weight classes, different competitive levels (Club, Area, National, International) and age at which RWL was first used (9-11, 12-14, 15-17, 18-20, $21+)$. The Bonferroni post hoc test was used to establish whether there were any significant differences between groups ${ }^{20}$. Analysing the weight classes, heavy weight athletes (females: $78+\mathrm{kg}$, males: $100+\mathrm{kg}$ ) were excluded ${ }^{14}$, since they do not regularly partake in RWL. The Chi-squared test examined differences in prevalence of RWL (nominal/ordinal data) between two or more independent variables (including gender, different competitive levels and different weight classes). All analyses were performed using the statistical package IBM SPSS Statistics for Windows (SPSS Inc., Chicago, IL, USA) version 22 , and the alpha level was set at $5 \%$.

\section{Results}

A total of 255 participants completed the questionnaire, with 235 completing the online questionnaire and 20 
Table III. Demographic and anthropometric characteristicsof the Judokas (Mean \pm SD).

\begin{tabular}{llll}
\hline Variable & Males & Females & Total \\
\hline $\mathbf{N}$ & 189 & 66 & 255 \\
Age (years) & $29.6 \pm 12.1$ & $24.0 \pm 5.8$ & $28.1 \pm 11.1$ \\
${\text { Body Mass }(\mathbf{k g})^{\star \star}}_{\text {Height }(\mathbf{c m})^{\star *}}^{80.6 \pm 16.6}$ & $176.3 \pm 7.8$ & $65.2 \pm 10.2$ & $76.6 \pm 16.6$ \\
\hline
\end{tabular}

** Significant difference between males and females (using independent sample t-test) $(p<0.01)$

Age (years): t:3.58 df:253 $p<0.01$

Body mass $(\mathrm{kg}): \mathrm{t}: 7.04 \mathrm{df}: 253 \mathrm{p}<0.01$

Height (cm): t:10.91 df:253 p<0.01
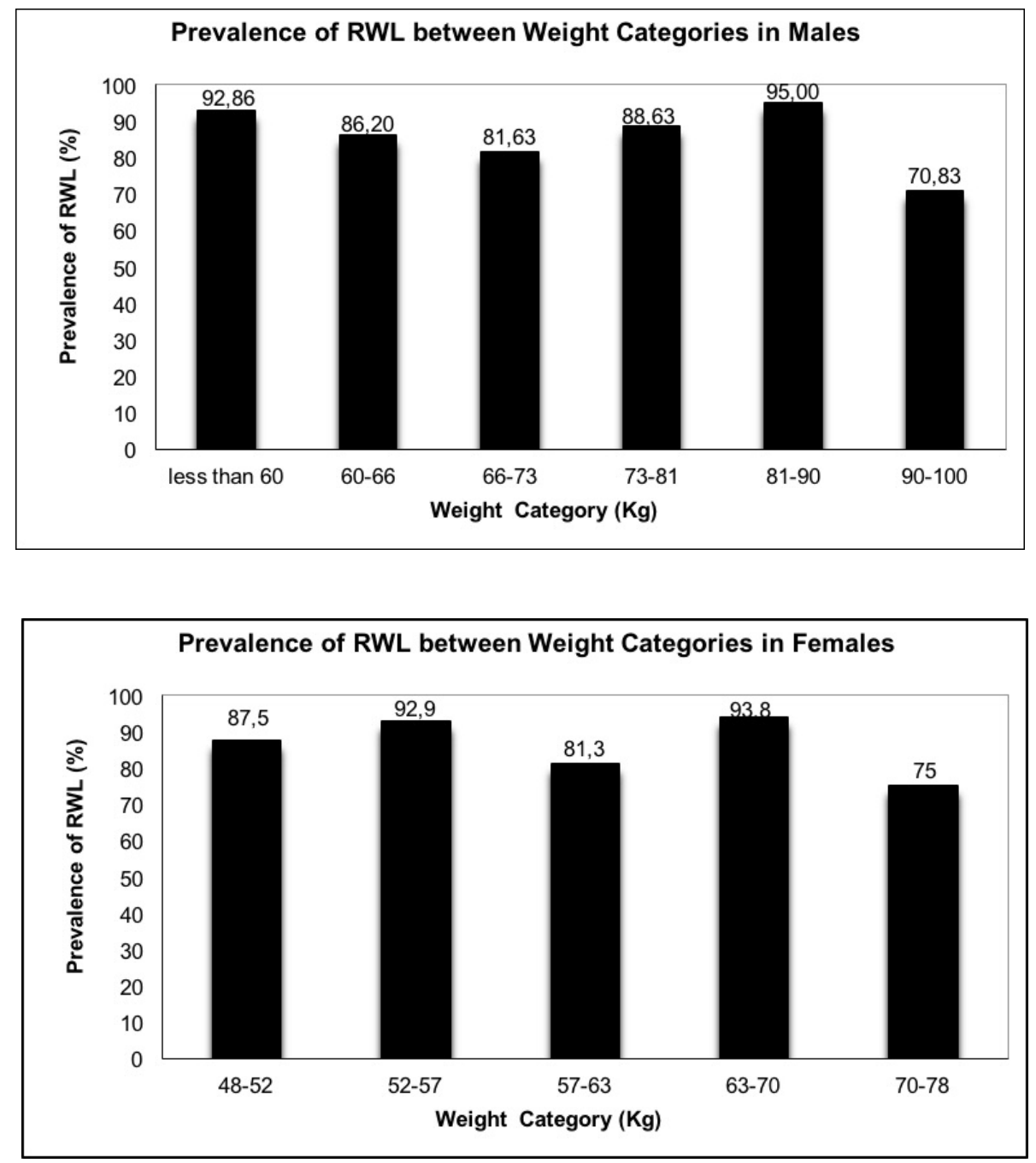

Figure 2. Prevalence of RWL by weight category in males. Heavy weights excluded. NS: P>0.05 (Chi-squared test: $\mathrm{p}=0.50$ ).
Figure 3. Prevalence of RWL by weight category in females. Heavy weights excluded. NS: P>0.05 (Chi-squared test: $\mathrm{p}=0.63$ ). using the paper questionnaire. $80 \%$ of judokas responded to the paper questionnaire, with the main reason for refusal being a lack of time.

\section{Demographics}

The gender breakdown was $75 \%$ males $(n=189)$ and $25 \%$ females $(n=66)$ with an age range of 18 to 67 years. Significant differences were found in age $(p=0.00)$, body mass $(p=0.00)$ and height $(p=0.00)$ between male and females (Tab. III).

\section{Prevalence}

The prevalence of RWL in males was $84.1 \%$ and for females was $84.8 \%$. The Chi-squared test revealed no statistical difference $(p=0.89)$ between them. 
Prevalence, techniques and knowledge of rapid weight loss amongst adult british judo athletes: a questionnaire based study
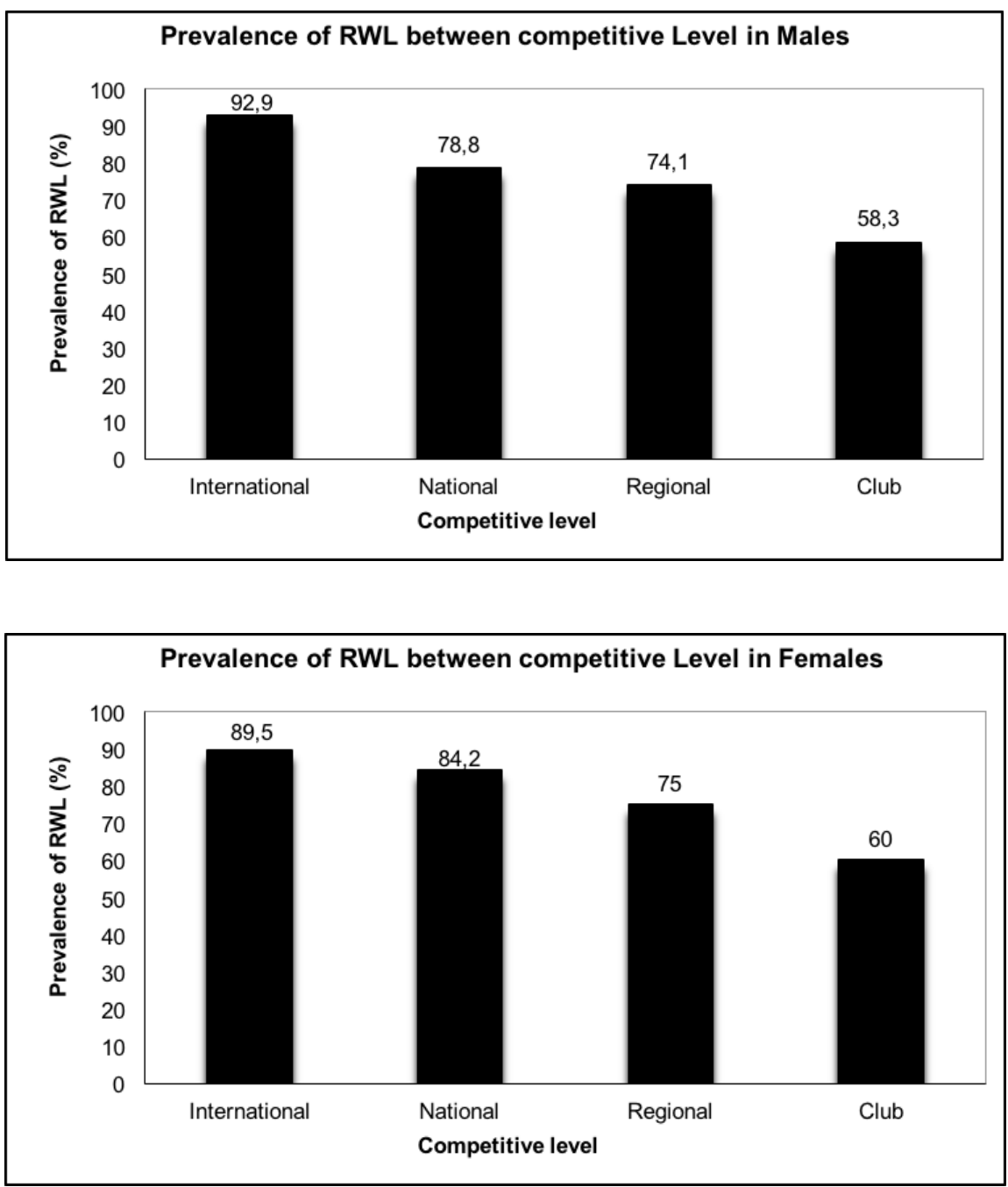

Figure 4. Prevalence of RWL between competitive levels in Males. ${ }^{*} \mathrm{P}<0.05{ }^{* *} \mathrm{P}<0.01$ (Chi-squared test).

International vs National: $\mathrm{p}=0.012$

International vs Regional: $\mathrm{p}=0.012$

International vs Club: $p=0.003$

Figure 5. Prevalence of RWL between competitive levels in females. NS: $P>0.05$ (Chi-squared test: $\mathrm{p}=0.34)$.

Table IV. Weight loss methods reported by judo athletes $(n=215)$.

\begin{tabular}{|c|c|c|c|c|c|}
\hline Methods & Always (\%) & Sometimes (\%) & $\begin{array}{l}\text { Almost Never } \\
(\%)\end{array}$ & Never (\%) & $\begin{array}{l}\text { Don't Use } \\
\text { Anymore (\%) }\end{array}$ \\
\hline Gradual Dieting* & 34.90 & 35.29 & 3.53 & 9.02 & 1.57 \\
\hline Skipping 1 or 2 meals & 14.90 & 28.24 & 21.96 & 16.86 & 2.75 \\
\hline Fasting $^{\star *}$ & 13.3 & 21.57 & 16.86 & 28.24 & 4.31 \\
\hline Restricting Fluid Intake & 25.10 & 32.94 & 9.02 & 14.9 & 2.35 \\
\hline Increase exercise & 40 & 32.16 & 6.6 & 3.53 & 1.96 \\
\hline $\begin{array}{l}\text { Training in heated } \\
\text { rooms }\end{array}$ & 10.98 & 20.00 & 15.69 & 35.30 & 2.35 \\
\hline Saunas & 13.73 & 22.35 & 12.55 & 31.76 & 3.92 \\
\hline $\begin{array}{l}\text { Training with } \\
\text { rubber/plastic suits }\end{array}$ & 12.55 & 16.47 & 10.20 & 40.39 & 4.31 \\
\hline $\begin{array}{l}\text { Using winter plastic } \\
\text { suits without exercise }\end{array}$ & 3.92 & 9.41 & 11.37 & 53.72 & 5.88 \\
\hline
\end{tabular}


There were no statistical differences in RWL prevalence between weight classes in either males $(p=0.50)$ (Fig. 2) or females ( $p=0.63$ ) (Fig. 3). The highest prevalence of RWL for males occurred in the $81-90 \mathrm{~kg}$ weight class (95.0\%) and for females occurred in 63-70 kg weight class $(93.8 \%)$.

Figure 4 and 5 show the trend of increased prevalence of RWL as competitive level increases in males and females. This was statistically significant when comparing international judokas to national $(p=0.012)$, regional $(p=0.012)$ and club level $(p=0.003)$ in males. In females, there were no significant differences in RWL prevalence between competitive levels $(p=0.34)$.

\section{Methods and Quantity of RWL}

Males and female judokas underwent a mean RWL of $2.4 \mathrm{~kg}$, with most athletes losing weight 5 times a season. Table IV shows the most common methods to achieve weight loss were "gradual dieting", "fluid restriction" and "increased exercise". Furthermore "laxatives", "diuretics", "diet pills" and "vomiting" were amongst the least common methods used by athletes.

\section{Knowledge Score}

The mean score for knowledge was 6.1 for males and 6.5 for females (out of 10). The best-answered questions were regarding injury risk (78\% correct) and reaction time (76\% correct). The worst answered questions were regarding heart function (57\% correct), bone density (50\% correct), and hormone imbalance (57\%).

\section{RWLB score}

The unpaired T-test showed that there was no significant difference in RWLB scores between males and females [t $(213)=-0.19, p=0.85]$ and between "high knowledge athletes" and "low knowledge athlete" [t $(49)=-0.90, p=0.37]$.

Table $V$ shows that there were no statistical differences in RWLB scores between different weight classes in both males $(p=0.15)$ and females $(p=0.93)$. There were significantly higher $(p=0.014)$ RWLB scores in different competitive levels in males. The post-hoc test revealed this to be between international athletes compared to regional athletes. There were no significant differences $(p=0.20)$ in RWLB scores between competitive levels in the female population. The age when RWL began had a significant effect $(p=0.000)$ on RWLB score in males, but not in females. The post hoc test showed that male judokas who began RWL between "9-11" and "12-14" years had significantly higher RWLB scores than those who began RWL between "18-20" and "20+" years.

\section{Discussion}

The main aim of this study was to identify the trends of RWL within the adult British judo population. To our knowledge, this is the first study of its kind analysing the weight loss behaviour of British judokas.

The prevalence of RWL amongst UK adult judokas was $84 \%$. This high prevalence is similar to a previous study ${ }^{14}$ conducted in Brazil (89\%). When compared to other combat sports (wrestling, jujitsu and taekwon$\mathrm{do}^{3,5,13}$ ), the prevalence of RWL in judo is much higher. A possible explanation is that judokas report that RWL gives them a psychological advantage over their opponent ${ }^{12}$. Moreover, because there were no differences in RWL prevalence among different genders/ weight class and since coaches/fellow judokas are the most influential in one's RWLB ${ }^{14}$, this suggests that a widespread RWL culture exists amongst British judokas.

In this study, most athletes lost an average of $2.4 \mathrm{~kg}$ about 5 times a season, which is consistent with the studies ${ }^{5,14}$ conducted in Brazil. The approaches to RWL were also similar to other combat sports ${ }^{5,13}$ with increased exercise, fluid restriction and gradual dieting being the main methods to reduce weight. Interestingly, the more severe methods of RWL (e.g. vomiting, laxatives, diuretics) were used much often less in the UK compared to other countries ${ }^{5,14}$. This could be due to the IJF ruling regarding unannounced weighing on the day of competitions ${ }^{15}$. This is because judokas are un-

Table V. ANOVA test results comparing mean RWL scores in different weight classes, competitive level and age RWL began for males and females.

\begin{tabular}{llll}
\hline Comparing RWL between: & F & DF & p-value \\
\hline Weight classes: Males & 1.66 & 5 & 0.15 \\
Weight classes: Females & 0.26 & 5 & 0.93 \\
Competitive level: Males & 3.64 & 3 & $\mathbf{0 . 0 1 4 ^ { \star }}$ \\
Competitive level: Females & 1.62 & 3 & 0.20 \\
Age RWL began: Males & 7.70 & 4 & $\mathbf{0 . 0 0 0 ^ { \star * }}$ \\
Age RWL began: Females & 2.42 & 4 & 0.06 \\
\hline
\end{tabular}

${ }^{*} p<0.05^{* *} p<0.01$

Competitive level in males: Bonferroni post-hoc test revealed significant difference in mean RWLB score between international and regional athletes only $(p=0.014)$.

Age RWL began in males: Bonferroni post-hoc test showed significant differences in mean RWLB score between age categories (years) "9-11" $\rightarrow$ "18-20" $(p=0.028) \&$ "20+" $(p=0.011)$

"12-14" $\rightarrow$ "18-20" ( $p=0.02) \& " 20+"(p=0.00)$ 
Prevalence, techniques and knowledge of rapid weight loss amongst adult british judo athletes:

a questionnaire based study

likely to undertake extreme RWL measures if their recovery period is considerably reduced.

Eating disorders are more common in females athletes, and a risk factor for developing them is partaking in weight-dependent sports ${ }^{21}$. However, no significant differences were found in RWL prevalence and RWLB between genders. This lack of difference might be explained by understanding that weight loss is goal-orientated towards making a weight class; meaning that weight reduction only occurs a limited number of times per season.

Furthermore, higher competitive levels had higher RWLB scores and prevalence in males. It can be noticed that aggressive RWLB is linked to greater desire for distinction that outweighs any health concerns ${ }^{12}$. However, no studies analysed whether RWL improves competitive success in judo, and the literature 22 surrounding the effects of RWL on power-related performance is controversial.

The male judokas who began RWL at younger ages had more aggressive RWLB in later life. This trend was also shown in studies assessing wrestlers ${ }^{3}$. Probably, those exposed to repeated bouts of aggressive weight loss from an early age normalise this behaviour, and thus attempt more extreme methods ${ }^{14}$. Furthermore, these athletes are more likely to compete at the elite level as adults as a consequence of greater experience and more coaching, and international level athletes have more aggressive RWLB ${ }^{14}$. Therefore, targeting young athletes could be an effective way of reducing dangerous RWL in adults in the future.

Educational programmes ${ }^{2}$ to reduce the prevalence of RWL have shown that judokas lack knowledge about RWL. In the present study, the average knowledge score was 6.2 (out of 10), suggesting that judokas have a moderate understanding of RWL. Furthermore, no significant difference in the RWLB scores between "low knowledge athletes" and "high knowledge athletes" were found. However, the knowledge section of the questionnaire was not tested for external validity, and therefore it may have been inadequate in discriminating "high" and "low" knowledge athletes, thus explaining these results. Furthermore, the closed questions questionnaire offers limited opportunity to assess knowledge, and is subjected to questionnaire bias. The results did, however, evidence that knowledge of judokas was particularly lacking regarding the physiological effects of RWL. Educational programmes reduce unhealthy behaviours (e.g. smoking and $\operatorname{diet}^{23}$ ), and therefore education may still be advantageous in reducing harmful RWL in judo.

From a clinical perspective, the new IJF ruling change regarding the weigh-in in international competitions is a positive step in reducing RWL, since this practice is most prevalent at the elite level. Stricter ruling with reduced recovery time between weigh-in and first bout has been shown to improve RWLB in American college wrestling ${ }^{11}$, and therefore should also be implemented in all levels of judo competition. Furthermore, since the age at which RWL first began is linked to aggressive RWLB in later life, youth athletes should also be targeted through reduced recovery times post weigh-in and random weigh-ins on competition day. Additionally, implementing educating strategies for judokas on the physiological harms of RWL and those most influential (coaches and colleagues ${ }^{14}$ ) to discourage this practice is still recommended to reduce the widespread RWL culture.

The main limitation of this study lies in the sampling method. The majority of questionnaires were completed online, and therefore response rates could not be calculated. Consequently, the results may be skewed, as only athletes who undertook RWL may have chosen to complete the questionnaire. Furthermore some judokas may have been omitted (those less adept with technology e.g. older population). The convenience sample was not stratified to randomly select participants from different parts of the UK, and so caution must be taken, as the findings should not be extrapolated to the whole British judoka population. However, response rates via online questionnaires remain high, and are efficient ways of obtaining large samples ${ }^{24}$. Moreover, amongst the judokas offered the paper questionnaire, there was a high acceptance rate, suggesting that athletes were willing to complete it. Also, the results were consistent with previous studies, suggesting that the use of a nonprobability sample impacted our findings only minimally. While significant trends for males were exhibited, they were not present in females. This could be a valid result, but it should be kept in mind that there were relatively few female judokas $(n=66)$, and consequently the female British judoka population may have not been accurately represented.

Another limitation is the use of self-reported questionnaire, which can introduce subjective self-assessment and recall bias. Nevertheless, this inaccuracy is minimised, through the use of the externally validated questionnaire ${ }^{17}$. Also, additional factors which can directly affect weight loss (medication ${ }^{25}$, supplements ${ }^{12}$ and medical conditions) were not evaluated in this questionnaire, and their inclusion could be considered for similar studies in the future.

Finally knowledge was shown to have no impact on RWLB. However, this may result from the fact that the developed questions had not been extensively externally validated and tested for reproducibility. However, the knowledge aspect of the questionnaire should have no bearing on the remainder of the questionnaire, which had been independently validated ${ }^{17}$ and successfully used ${ }^{14}$.

\section{Conclusion}

The prevalence of RWL in British judokas is high, and independent of weight class or gender, but affected by competitive level in males, indicating a widespread RWL culture. British judokas tended to have moderate knowledge regarding RWL, but did not know much regarding its physiological effects. Furthermore, internationally ranked male judokas and those male judokas who began RWL earliest were the most likely to use the most aggressive RWL measures.

These findings have immediate clinical bearings: they 
will assist in targeting the problem of dangerous RWL. Recent weigh-in changes at elite level should be complemented with similar changes at the amateur and youth level. Additionally, education of athletes on the physiological harms of RWL is recommended.

\section{References}

1. Federation IJ. Judo Corner- Judo History [Available from: http://www.intjudo.eu/Judo_Corner

2. Artioli GG, Franchini E, Nicastro H, Sterkowicz S, Solis MY, Lancha $\mathrm{AH}$. The need of a weight management control program in judo: a proposal based on the successful case of wrestling. J Int Soc Sports Nutr. 2010;7:15.

3. Kiningham RB, Gorenflo DW. Weight loss methods of high school wrestlers. Med Sci Sports Exerc. 2001;33(5):810-813.

4. Kazemi M, Shearer H, Choung YS. Pre-competition habits and injuries in Taekwondo athletes. BMC Musculoskelet Disord. 2005;6:26.

5. Brito CJ, Roas AF, Brito IS, Marins JC, Cordova C, Franchini E. Methods of body mass reduction by combat sport athletes. Int J Sport Nutr Exerc Metab. 22. United States. 2012: 89-97.

6. Prouteau S, Pelle A, Collomp K, Benhamou L, Courteix D. Bone density in elite judoists and effects of weight cycling on bone metabolic balance. Med Sci Sports Exerc. 2006;38 (4):694-700.

7. Maffulli N. Making weight: a case study of two elite wrestlers. Br J Sports Med. 1992;1992 Jun;26(2):107-110.

8. Jlid M, Maffulli N, Elloumi M, Moalla W, Paillard T. Rapid weight loss alters muscular performance and perceived exertion as well as postural control in elite wrestlers. J Sports Med Phys Fitness. 2013 Dec;53(6):620-627.

9. Allen TE, Smith DP, Miller DK. Hemodynamic response to submaximal exercise after dehydration and rehydration in high school wrestlers. Med Sci Sports. 1977;9(3):159-163.

10. Choma CW, Sforzo GA, Keller BA. Impact of rapid weight loss on cognitive function in collegiate wrestlers. Med Sci Sports Exerc. 1998;30(5):746-749.

11. Oppliger RA, Utter AC, Scott JR, Dick RW, Klossner D. NCAA rule change improves weight loss among national championship wrestlers. Med Sci Sports Exerc. 38. United States. 2006:963-970.
12. Pettersson S, Ekstrom MP, Berg CM. Practices of weight regulation among elite athletes in combat sports: a matter of mental advantage? J Athl Train. 2013;48(1):99-108.

13. Coufalová $K$, Prokešová $E$, Malý $T$. Body weight reduction in combat sports. Science Of Martial Arts. 2013;9(4):267-272.

14. Artioli GG, Gualano B, Franchini E, Scagliusi FB, Takesian M, Fuchs M, et al. Prevalence, magnitude, and methods of rapid weight loss among judo competitors. Med Sci Sports Exerc. 2010;42(3):436-442.

15. (IJF) IJF. Sport Organisation Rules (SOR) Referee Rules 2014. Available from: http://www.intjudo.eu/upload/2013_12/ 03/138608201843375525/referee_rules_2014_2016_gb.pdf

16. Franchini E, Brito CJ, Artioli GG. Weight loss in combat sports: physiological, psychological and performance effects. J Int Soc Sports Nutr. 9. United States. 2012:52.

17. Artioli GG, Scagliusi F, Kashiwagura D, Franchini E, Gualano B, Junior AL. Development, validity and reliability of a questionnaire designed to evaluate rapid weight loss patterns in judo players. Scand J Med Sci Sports. 2010;20(1):e177-187.

18. Padulo J, Oliva F, Frizziero A, Maffulli N. Muscles, Ligaments and Tendons Journal - Basic principles and recommendations in clinical and field science research: 2016 update. MLTJ. 2016;6(1):1-5.

19. Manikandan S. Data transformation. J Pharmacol Pharmacother. 2010;1(2):126-127.

20. Platt R. ANOVA, t tests, and linear regression. Inj Prev. 1998;4(1):52-53.

21. Sundgot-Borgen J. Risk and trigger factors for the development of eating disorders in female elite athletes. Med Sci Sports Exerc. 1994;26(4):414-419.

22. Mendes SH, Tritto AC, Guilherme JP, Solis MY, Vieira DE, Franchini $\mathrm{E}$, et al. Effect of rapid weight loss on performance in combat sport male athletes: does adaptation to chronic weight cycling play a role? British journal of sports medicine. 47. England. 2013:1155-1160.

23. Amani R, Soflaei M. Nutrition education alone improves dietary practices but not hematologic indices of adolescent girls in Iran. Food Nutr Bull. 2006;27(3):260-264.

24. Hunter L. Challenging the reported disadvantages of e-questionnaires and addressing methodological issues of online data collection. Nurse Res. 2012;20(1):11-20.

25. Antel J, Hebebrand J. Weight-reducing side effects of the antiepileptic agents topiramate and zonisamide. Handb Exp Pharmacol. 2012(209):433-466. 